\title{
A Clinical study of Retinal vein occlusions and Management
}

\author{
Dr.D. Bhima Sankar Babu M.S(ophthal) ${ }^{1,}$ Dr.M. Vijaya Leela M.S(ophthal) \\ ${ }^{1}$ Asst.Professor Of Ophthalmology Osmania Medical College/Sarojini Devi Eye Hospital \\ ${ }^{2}$ Asst.Professor Of Ophthalmology Osmania Medical College/Sarojini Devi Eye Hospital
}

\begin{abstract}
RVO in 50 patients were studied in this series giving special consideration to high risk factors and manifestations, results of various modalities of management and eventual complications.
\end{abstract}

Aims and objectives of the study :

1. To study the prevalence of disease

2. To assess risk factors for $R V O$

3. To study the results of various modalitiesof treatment

Inclusion criteria :

Criteria For Crvo : flame shaped,dot or punctuate retinal hemorrhages or both in all four quadrants of the retina, dilatation and increased tortuosity of retinal veins and optic disc swelling with or without macular edema.

Hcrvo : same as CRVO but involving either superior or inferior half of the retina only

Brvo :

Initially : either flame shaped,dot or punctuate retinal hemorrhagesin the distribution of occluded branch dilataion and tortuisity of the occluded vein.

Later : Criteria expanded to include cases in whom retinal hemorrhages had resolved and new vessels or collaterals have developed.

Investigations related to various risk factors were done in accordance with our proforma.The course of the disease was monitored through out study period and appropriate intervention in individual cases was done based on its necessity;

Exclusion criteria : Those with associated diabetic retinopathy changes on fundus examination.

\section{Materials And Methods}

Our study named "A CLINICAL STUDY OF RETINAL VEIN OCCLUSIONS AND MANAGEMENT" deals with analysis of various aspects of the three main morphological types of retinal vein occlusions. This study is conducted on 50 eyes of 50 patients who presented to the sarojini devi hospital hyderabad. Of these 50 eyes 13 cases are CRVO ,2 cases are HRVO ,36 cases of BRVO.In this series only unilateral effected cases and patients with more than 50 years were included.

II. Materials :

Patients attending our OPD at sarojini devi eye Hospital who are diagnosed to have RVO .Totally 50 eyes of 50 patiemts were studied.

1. Epidemiological characteristics

\section{Discussion}

2. clinical features

3. treatment

\section{Epidemiological characteristics:}

Prevalence of RVO: Prevalance of RVO among the patients attending out patient ophthalmology department at Sarojini devi eye hospital ,hyderabad is $0.68 \%$.This prevalence results were consistent with prevalence of Baever Dam eye study conducted by Klein.R where prevalence was $0.6 \%$

Incidence of various types of RVO : In this study incidence of BRVO was 70\%,HCRVO was $26 \%$.These results are concordant with results of Blie Australian conducted by Michell.p et all.(BRVO$69.5 \%, \mathrm{HCRVO}-5.1 \%, \mathrm{CRVO}-25 \%) .{ }^{60}$

Age distribution: Mean age groupbfor CRVO in this study was 66 yrs and the mean age for BRVO was 65 years. The over all mean age group was 67 years.

Sex distribution: In this study we found a slight male preponderance for both CRVO and BRVO with approximately $54 \%$ incidence respectively .Higher prevalence of RVO in male sex is documented by Hayreh 
.S.S of 122 eyes with RVO in 108 patients.Explanation for increased incidence of RVO in males is on hormonal basis.Higher haemotocrit in males could act as contributory factor in elevating blood viscosity and producing $\mathrm{RVO}^{9}$.

Laterality: This study is dealing with unilateral cases of RVO.Out of 13 cases of CRVO that was studied,5(38.46\%) involved the right eye and 8(61.53\%) involve the left eye.

Branch involved in CRVO: No significant comment can be made here since the number of HCRVO studied was very less.

Branch involved in BRVO:Superotemporal is the commonest site of occlusion in BRVO.As is already known this was corroborated well in this study ST-BRVO forming about $57.14 \%$ of the total cases followed by IT-BRVO which formed about $28.58 \%$.There was a single case of Sn quadrant being involved, while 4 casea had a macular BRVO.

Place Of Study : The study was carried out at Sarojini devi eye hospital, hyderabad

Study Design : Retrospective study

\section{Methods}

This study was performed on analysis of prevalence, risk factors ,clinical presentation,management and complication of these 50 patients

Initially detailes proforma was prepared including all the factors intended in the study.A detailed case history including age, gender,occupation, economic status and predisposing factors like hypertension, diabetes and ischaemic heart disease and renal disease.History of smoking was recorded.A detailed interrogation was made to elicit the other signs and symptoms to exclude other ocular conditions like POAG which is a known risk factor.Thorough examination consisted of visual acuity recording, external examination and slit lamp examination,AS examination and PS examination with direct and indirect ophthalmoscopy and $90 \mathrm{D}$ examination,fundus photographs are taken and fundus camera and IOP was recorded.

\section{Results}

1. In this study 13 eyes with CRVO , 2 with HCRVO and 35 cases with BRVO were considered.Thus CRVO consists of 26 percent ,HCRVO 4 percent and BRVO 70 percent of the total RVO patients.

2. Mean age in the CRVO group was 66 years, HCRVO 66 years and BRVO WAS 65 years.

3. Gender wise incidence for both CRVO and BRVO had slight male preponderance with approximately 53.85 percent and 54.28 percent incidence respectively.Thia is probably due to the relatively higher haemotocrit in males.

4. Among BRVO cases, superotemporal vein occlusion (57.14\%) was commonest, $28.58 \%$ cases were infero temporal , 2.86 percent were supero nasal.(only 1 case) and $11.42 \%$ had macular BRVO .There was no case infero nasal BRVO.

5. Among CRVO cases 53.84 cases were hypertensives, $15.32 \%$ were diabetics. $46.15 \%$ patients had high serum cholesterol, but just $15 \%$ ( 2 in number )were IHD patients and 2 patients had an elevated IOP more than $20 \mathrm{~mm}$ of HG.There was one patient with retinal disease.There was 1 patient with renal disease.Smoking is present in 23.07 percent.

6. In the BRVO group of 35 patients $65.71 \%$ were hypertensives and $23.85 \%$ were diabetics $(71 \%)$.Serum cholesterol was elevated in $28.57 \%$ and only $8.57 \%$ ( 3 patients ) were having IHD.11.42 \%had a high IOP.Smoking is present in $25.71 \%$.

7. Statistics of the HCRVO group cannot be considered very significant because only 2 patients with HCRVO were included in the study.One was hypertensive, one had serum cholesterol more than $200 \mathrm{mg} \%$.

8. Only $31 \%$ of CRVO cases and $40 \%$ of BRVO cases presented within 1 month of onset of disease.The rest had a variable duration of disease upto 1 year before they presented to us.

9. $4 \%$ of cases CRVO and $50 \%$ cases of HCRVO presented with visual acuity of less than $6 / 60$ while among BRVO cases this number was 49\%.FFA findinds were as follows : 4 patients fo CRVO HAD CNP wgere 9 patients had ME.Both HCRVO patients had ME.Among BRVO cases 3 had CNP $<5$ DD , 1 had CNP $>5$ DD , 4 had NVD/NVE while 20 had ME.

10. Inthis study $46.15 \%$ of CRVO and $42.85 \%$ of BRVO cases had normal axial length.The association of hypermetropia with CRVO is seen in $23.07 \%$ and BRVO in $28.57 \%$ which is not signoificant in this study.

11. 9 od 13 cases of CRVO show evidence of macular edema , 4 cases shows capillary non perfusion.

12. This study of 35 cases of BRVO, 4 cases showed areas of capillary non perfusion alone without any neovascularisation, 1 of whoch had CNP more than 5 DD.4 cases showed evidence of NVD/NVE, while 20 cases had macular edema shown by late staining of macula.2 cases showed both neovascularisation and macular edema.All BRVO cases revealed the sight of venous occlusion with dilation of vein distal to the AV crossing and late staining of the vessels.FFA was not conclusive in 2 cases because of the view due to extensive hemorrhages. 
13. Out of 13 cases of RVO , 9 cases are treated with repeated injections of intravitreal avastin and followed up 2 cases are treated with PRP and intravitreal avastin ,remaining 2 cases were managed with general medical treatment and then followed up.

Right eye :Infero temporal brvo with macular edema with grade 2 hypertensive retinopathy Left eye: grade 2 hypertensive retinopathy
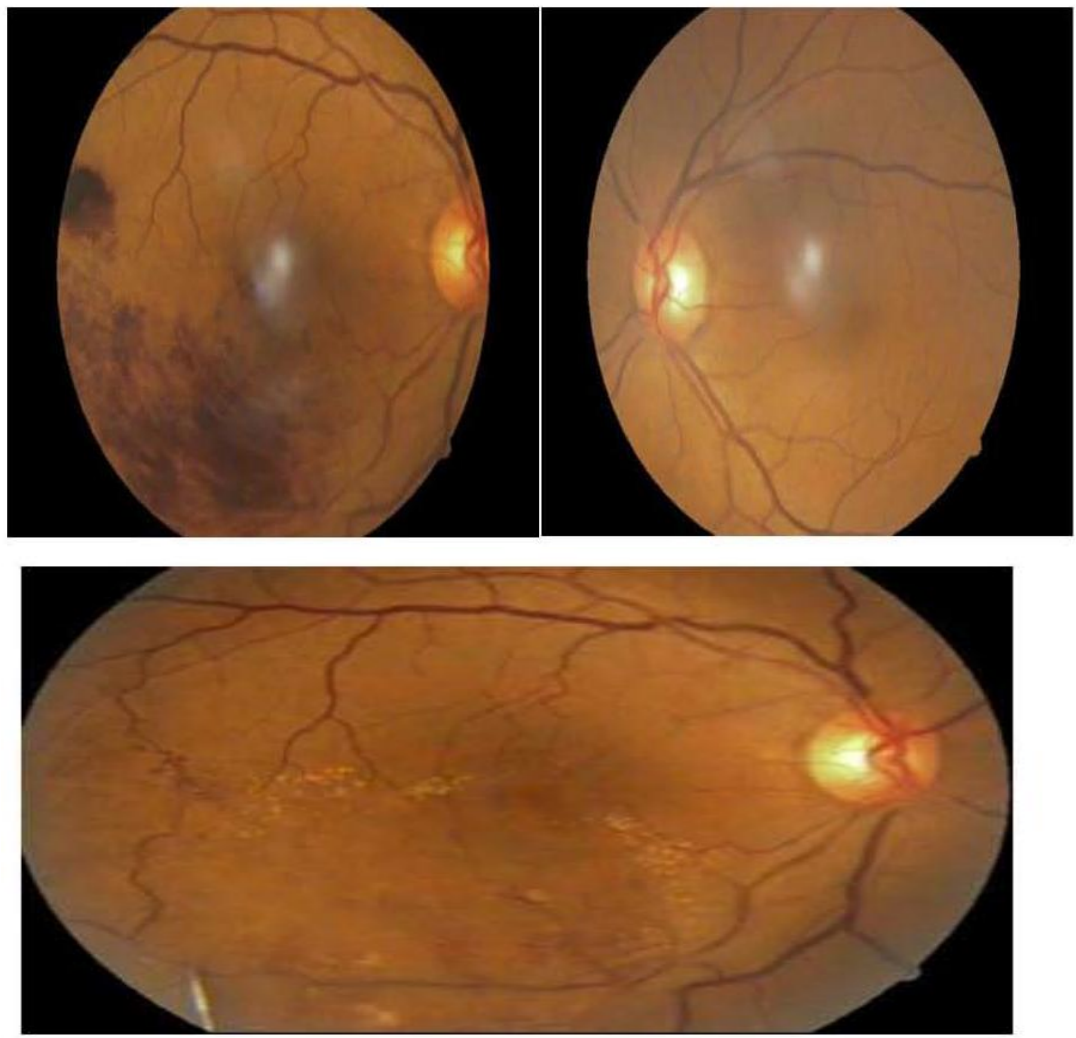

Right eye follow up picture after intravitreal avastin

\section{Treatment protocol for RVO:}

Treatment of underlying systemic condition like hypertension, diabetes mellitus, ihd and renal disease based on physician opinion.Medical management given to all patients consists of anti-oxidants and tablet vitamin $C$ as supportive therapy.Patients with cvs risk factors were treated with tablet trental(pentoxyphyline) $400 \mathrm{mg}$ BD for 3 months.

CRVO with macular edema-intra vitreal avastin injection with monthly follow up and repeated based on the visual acuity and response of the macular edema to the injections

\section{Conclusions}

\section{The following conclusions could be derived from this study:}

1. Hypertension was proven to have a strong association with both CRVO \& BRVO,definitely much stronger than diabetes.smoking and hyperlipidaemia was proven to have association with RVO.also,hypertension formed a higher risk for BRVO $(65.71 \%)$ in this study than CRVO(53.84\%).

2. slight male preponderance was found in both CRVO \& BRVO which could be possibly be explained by the raised haematocrit value in males.

3. Retinal vein occlusion were common in elderly age group.

4. Reduced axial length \& hypermetropia were not significantly associated with RVO in this study.

5. Serum cholesterol more than $200 \mathrm{mg} \%$ was found be a significant risk factor for RVO and patients may be benefited by dietary changes and appropriate drug therapy.

6. Statistics of the HCRVO group cannot be considered very significant because only two patients were included.

7. Ischaemic CRVO has a low presenting visual acuity $<3 / 60 \&$ poor prognosis for vision as well.Non iscaemic CRVO on the other hand has an overall better presenting visual acuity and the macular oedema 
when managed with intravitreal Avastin gives an improvement in the visual acuity.FFA is an important diagnostic modality especially to find out areas of peripheral neovascularization and iscaemic areas as suggested by capillary drop outs which may be missed out on ophthalmoscopy and fondus photography.Hence it is a must in all RVO cases.

8. In this study all VSR tyupe of CRVO cases that showed macular oedema has a complication treated with repeated intravitreal Avastin injections and the final visual acuity improved (6 of 9) for atleast remained stationary 3 cases of CRVO have a macular oedema,but definitely did not deteriorate.In BRVO casese with macular oedema treated with intravitreal Avastin injections.most of them show either improvement of visual acuity or stationary vision.Hence it can be a good option of treatment significant benefit to the patients.

9. However laser photocoagulation either panretinal as done in CRVO cases or sectoral as in BRVO cases cannot be said to have a consistently good response in terms of visual acuity,but they show good response in reduction of neovascularization either in anterior segment or posterior segment.

10. Patients of BRVO with significant ischaemia presenting with initial vision of $3 / 60$ or less (poor vision) $\mathrm{BRVO}$ cases are unlikely to improve as evidence in poor vision in BRVO cases.

11. Due to small number of HCRVO cases in this study we could not assess the ischaemic HCRVO versus VSR type HCRVO.but our cases were VSR type with macular oedema \& responded well significantly well to treatment given.

12. In case of BRVO present with macular oedema,treatment with repeated injections of intravitreal Avastin shows significant improvement in visual acuity(11 of 19).persistent macular oedema treated with grid LC shows improvement (4 of 5) or atleast did not show any deterioration.

13. Finally I would sum up this study by concluding that beware of two H's in RVO: Hypertension, hypercholeseteraelemia

14. The scenario of BRVO is different though,with variable results and a good chance for visual improvement if the patient presents with moderate visual acuity better than $6 / 60$.

[1]. Eugene wolf anatomy of the eye and orbit $7^{\text {th }}$ edition 1976

\section{References}

[2]. Duke elder-Diseases of the retina vol x 1967

[3]. Parsons disease of the eye $20^{\text {th }}$ edition

[4]. Yanoff and Duker ophthalmology $3^{\text {rd }}$ edition chapter 6,17

[5]. Jack.J.Kanski-clinical ophthalmology $6^{\text {th }}$ edition

[6]. Peyman-Principles and practice of phthalmology vol 21987

[7]. Stephen.J.Ryan-Medical retina vol 21989

[8]. Ocular pathology-greer $3^{\text {rd }}$ edition 1975 